\title{
Comparison of an Accelerometer-Based Portable Navigation System, Patient-Specific Instrumentation, and Conventional Instrumentation for Femoral Alignment in Total Knee Arthroplasty
}

\author{
Kohei Kawaguchi, MD, Kazuhiko Michishita, MD, Takeshi Manabe, MD, Yoshiyuki Akasaka, MD, \\ and Junya Higuchi, MD \\ Department of Orthopaedic, Japan Community Healthcare Organization, Yugawara Hospital, Kanagawa, Japan
}

\begin{abstract}
Purpose: The KneeAlign2 (KA2, OrthoAlign Inc.) accelerometer-based portable navigation system and patient-specific instrumentation (PSI; Signature, ZimmerBiomet) are widely used for ideal femoral component alignment in total knee arthroplasty (TKA). However, there has been no comparative study of the KA2 system, PSI, and conventional intramedullary instrumentation (CON). The purpose of this study was to compare the accuracy in achieving proper femoral component alignment and clinical features by using the KA2 navigation system, PSI, and CON.

Materials and Methods: We retrospectively compared the accuracy of femoral component alignment of 34 TKAs performed with the KA2 system for implantation of the femoral component, 32 TKAs with PSI, and 33 TKAs with CON.

Results: In the coronal plane, use of the KA2 system was more likely to result in optimal femoral component alignment than the CON and PSI ( $<<0.01$ ). In the sagittal plane, use of the KA2 system was more likely to result in optimal component alignment than PSI, but the difference between the KA2 and CON was insignificant.

Conclusions: The portable accelerometer-based KA2 navigation system enabled ideal femoral implantation in the coronal and sagittal planes, as compared to the PSI or CON.
\end{abstract}

Keywords: Knee, Arthroplasty, Navigation, Patient-specific instrumentation, Femoral component alignment

\section{Introduction}

It is generally recognized that the primary goal of total knee arthroplasty (TKA) is to restore a neutral mechanical axis and to align the components perpendicular to the mechanical axis of the leg in the coronal plane. Since malalignment in proximity to the

Received May 8, 2017; Revised (1st) June 13, 2017; (2nd) July 11, 2017; (3rd) July 18, 2017; Accepted July 24, 2017

Correspondence to: Kohei Kawaguchi, MD

Department of Orthopaedic, Japan Community Healthcare

Organization, Yugawara Hospital, 438 Miyakami, Yugawara, Kanagawa 259-0396, Japan

Tel: +81-465-63-2211, Fax: +81-465-62-3704

E-mail: kkohei0602@yahoo.co.jp

This is an Open Access article distributed under the terms of the Creative Commons Attribution Non-Commercial License (http://creativecommons.org/licenses/by-nc/4.0/) which permits unrestricted non-commercial use, distribution, and reproduction in any medium, provided the original work is properly cited. mechanical axis results in a higher failure rate, it is very important to accurately align the components as much as possible ${ }^{1,22}$.

The most common methods for alignment of the components in TKA are the intramedullary guide for alignment of the femur and the extramedullary guide for alignment of the tibia. However, these conventional methods have demonstrated a limited degree of accuracy for component placement ${ }^{3-5}$. In particular, the conventional intramedullary guide for distal femoral osteotomy $(\mathrm{CON})$ is less accurate than the extramedullary guide for proximal tibial osteotomy ${ }^{5}$ and results in greater blood loss because of injury to the intramedullary canal ${ }^{6,7)}$. Therefore, the inaccuracy of distal femoral osteotomy and risk of injury remain serious drawbacks. However, some useful devices were recently developed to increase the accuracy of implant alignment and to decrease the risk of injury to the intramedullary canal, such as computer-assisted navigation systems (CAS) with large consoles and patientspecific instrumentation (PSI). 
Several previous studies revealed the accuracy of implant alignment with the use of a large-console CAS and addressed several potential problems associated with large initial start-up costs, pin site locations, and large consoles ${ }^{8-11)}$. The KneeAlign2 (KA2; OrthoAlign Inc., Alisco Viejo, CA, USA) system is an accelerometer-based, portable, navigation device for TKA that combines the alignment accuracy of a large-console CAS with ease of use and convenience of conventional alignment methods. The KA2 system does not require the use of a large console or navigation arrays for registration and alignment feedback. Nam et al. ${ }^{12)}$ reported superior accuracy of the KA2 system, as compared to a large-console, imageless CAS.

Similarly, the use of PSI increases accuracy of implant alignment. Some previous studies revealed that PSI improved the accuracy of mechanical alignment restoration and component alignment, as compared to conventional instrumentation ${ }^{13-15)}$. However, some studies indicated that the alignment was not as accurate as was expected, additional time was needed for preoperative magnetic resonance imaging (MRI) or computed tomography (CT), and it was necessary to fabricate customized guides $^{16,17)}$.

Large-console CASs require large start-up capital costs, while the KA2 system and PSI provide a degree of familiarity to general surgeons and have lower initial costs. There is only one comparative study of the KA2 and PSI ${ }^{18)}$. Steinhaus et al. ${ }^{18)}$ reported there were no differences in coronal alignment, and PSI resulted in significantly more outliers in tibial sagittal alignment. Other than this, however, there has been no comparative study of the KA2 system and PSI to date. Therefore, the aim of this study was to investigate and compare the accuracy of the accelerometer-based KA2 navigation system, PSI, and CON to achieve appropriate positioning of femoral components.

\section{Materials and Methods}

We retrospectively reviewed the radiographic and operative results of 32 consecutive patients ( 6 males and 26 females) who underwent TKA performed using the KA2 system for distal femur resection and extramedullary devices for proximal tibial resection in our facility from June 2014 to June 2015. From October 2013 to July 2014, 31 consecutive patients (4 males and 27 females) underwent TKA performed using PSI for both distal femoral and proximal tibial resections. From November 2012 to June 2013, 32 consecutive patients ( 7 males and 25 females) underwent TKA performed using a conventional intramedullary rod for distal femoral resection with an extramedullary device for proximal tibial resection. Inclusion criteria for this study were a history of medial osteoarthritis, primary TKA with Biomet Vanguard PS (Zimmer, Warsaw, IN, USA) components, and a postoperative long-leg radiograph taken at our facility. Patients with rheumatoid arthritis, with valgus deformity and without a long-leg radiograph were excluded. We used several kinds of components from several manufactures and they had own ideal implant positions and rotations. So we excluded other components other than Vanguard PS. Additionally, this study needed a precise postoperative long-leg radiograph to get precise component alinement; therefore, we excluded patients who had a malrotated long-leg radiograph. As a result, we had to exclude 65 patients who underwent primary TKA from November 2012 to June 2015. This study was granted Institutional Review Board approval.

\section{Operative Techniques and Procedures}

All patients received Biomet Vanguard PS components. The surgical techniques were standardized apart from the use of femoral instruments for osteotomy. The KA2 system was used for distal femoral osteotomy. A display console and a reference sensor were used for distal femoral resection. The KA2 system is designed to determine the position of the cutting blocks in both the coronal (varus/valgus) and sagittal (flexion/extension) planes relative to the established femoral mechanical axis. We adjusted the position of the cutting block using the display console, which provides real-time feedback of the cutting guide orientation relative to the mechanical axis, and we aimed to set the guide perpendicular to the femoral mechanical axis in the coronal plane. In the sagittal plane, we aimed to set the guide perpendicular to the distal femoral anatomical axis to prevent anterior notching and set $3^{\circ}-4^{\circ}$ of flexion relative to the sagittal mechanical axis considering femoral bowing. Tibial osteotomy was performed with an extramedullary guide; the same as with the conventional method. The KA2 system was not used for proximal tibial osteotomy because we needed more skin incision to set the guide.

Biomet Signature PSI technology (Zimmer) was used for the PSI group. This process began with preoperative MRI or CT of the entire lower limb in accordance with the manufacturer's protocol. Imaging data were then uploaded into primary postprocessing software (Mimics Innovation Suite; Materialise NV, Leuven, Belgium) to create a computer-generated preoperative plan according to the surgeon's preferences. Our preferable femoral component alignment was perpendicular to the femoral mechanical axis in the coronal plane and perpendicular to the femoral anatomical axis in the sagittal plane. Our preferable tibial component alignment was $90^{\circ}$ to the mechanical axis and the 
sagittal alignment was $3^{\circ}$ of the posterior slope. The surgeon assessed the preoperative plan with the option to change multiple option variables, including implant size, alignment, and resection level. Once the plan was approved, the femoral and tibial guides were manufactured by Materialise to fit the unique anatomy of each patient to guide surgical bone resection. The custom pinning guides were applied to the distal femur and proximal tibia before removal of osteophytes. After inserting the pins, conventional cutting jigs were placed in position to make the bone cuts. We used both CT-based and MRI-based Signature customized planning system. Only five patients could not have preoperative MRI, because they had pacemakers or metallic devices. In the CON group, intramedullary femoral jigs were used. We decided valgus angles between an anatomical femoral axis and a mechanical femoral axis from evaluating entire lower limb preoperative radiographs.

For proximal tibial osteotomy, an extramedullary jig was used in the KA2 and CON groups, while a PSI tibial guide was used in the PSI group. Postoperative tibial component positions in the coronal and sagittal planes are shown in Fig. 1.

Clinical preoperative data was collected retrospectively. Patient
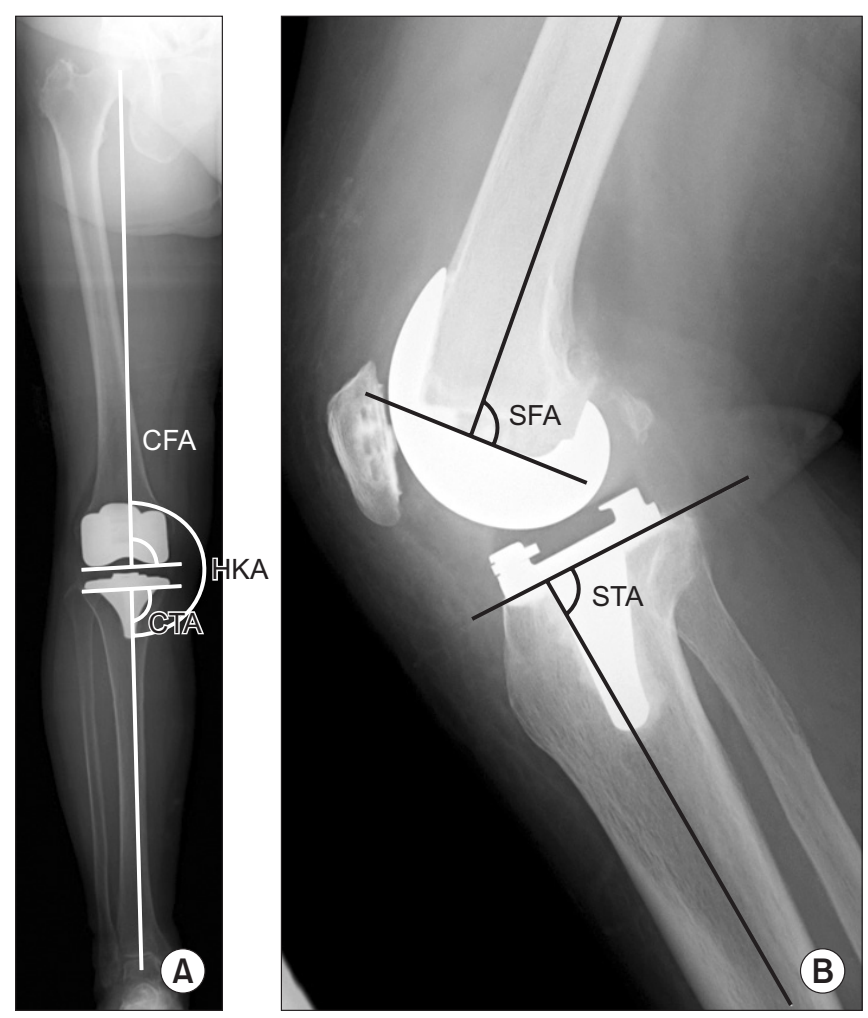

Fig. 1. (A) Anterioposterior radiograph of the entire lower limb after total knee arthroplasty. (B) Lateral radiograph of the knee. CFA: coronal femoral angle, HKA: hip-knee-ankle angle, CTA: coronal tibial angle, SFA: sagittal femoral angle, STA: sagittal tibial angle. characteristics, including age, sex, and body mass index (BMI), were recorded. Clinical assessment included the Knee Society score (KSS), consisting of two subscales (the knee and function scores), and range of motion (ROM), which were recorded both before and 1 year after TKA. We also recorded the surgical duration (skin to skin). Additionally, we estimated blood loss according to method described by Nadler et al. ${ }^{19}$. Preoperative patient characteristics of the three groups (age, sex, BMI, side, femoral tibial angle, ROM, and KSS) are shown in Table 1. There were no significant differences in preoperative characteristics among the three groups.

\section{Outcome Measurements}

Anteroposterior (AP), lateral, and AP entire lower limb postoperative radiographs were obtained before surgery and 1 year after TKA. The entire lower limb view was used to assess the mechanical axis and individual component positions in the coronal plane. The lateral view was used to assess the component positions in the sagittal plane. The femoral mechanical axis was first determined by drawing a line from the center of the femoral head (determined using a best-fit circle) to the intercondylar notch of the implant. For coronal mechanical alignment, the hipknee-ankle angle (HKA) was measured on the entire lower limb radiographs. The coronal femoral component angle (CFA) was defined as the angle between the femoral mechanical axis and the tangent formed by the distal femoral condyle. The coronal tibial component angle (CTA) was measured as the angle between the mechanical axis and the tibial plateau. The lateral component position was defined as the angle between the anatomical femoral axis or anatomical tibial axis in lateral short radiographs and the respective implant surfaces (sagittal femoral component angle

Table 1. Preoperative Patient Characteristics

\begin{tabular}{lcccc}
\hline \multicolumn{1}{c}{ Characteristic } & KA2 $(\mathrm{n}=32)$ & PSI $(\mathrm{n}=31)$ & CON $(\mathrm{n}=32)$ & $\mathrm{p}$-value \\
\hline Age $(\mathrm{yr})$ & $76.0 \pm 5.2$ & $74.2 \pm 8.0$ & $72.3 \pm 5.1$ & 0.059 \\
Sex $($ female:male $)$ & $26: 6$ & $27: 4$ & $25: 7$ & 0.196 \\
BMI $\left(\mathrm{kg} / \mathrm{m}^{2}\right)$ & $25.3 \pm 2.5$ & $25.9 \pm 3.2$ & $27.2 \pm 3.5$ & 0.057 \\
Preop HKA $\left(^{\circ}\right)$ & $167.2 \pm 4.8$ & $167.7 \pm 4.5$ & $168.1 \pm 6.1$ & 0.404 \\
Preop ROM $\left(^{\circ}\right)$ & $111.1 \pm 16.1$ & $114.8 \pm 17.4$ & $107.5 \pm 22.8$ & 0.158 \\
Preop KSS & $46.2 \pm 8.4$ & $48.8 \pm 3.8$ & $47.3 \pm 5.9$ & 0.266 \\
Preop Knee function & $42.9 \pm 21.3$ & $47.2 \pm 9.4$ & $44.3 \pm 12.9$ & 0.528 \\
score & & & &
\end{tabular}

Values are presented as mean \pm standard deviation.

KA2: KneeAlign2, PSI: patient-specific instrumentation, CON: conventional intramedullary instrument, BMI: body mass index, HKA: hipknee-ankle angle, ROM: range of motion, KSS: Knee Society score. 
[SFA], sagittal tibial component angle [STA] ${ }^{15}$ (Fig. 1). The component alignments were considered normal if they were within $\pm 3^{\circ}$ of the desired positions. Deviation of more than $3^{\circ}$ from the target in any plane was defined as an outlier. Radiographic measurements were performed twice at two different time points by two independent examiners (KK and JH). Intra- and inter-rater comparisons revealed measurement errors of less than one degree for all examined parameters.

\section{Statistical Analyses}

All data were collected and analyzed using the Excel statistics 2015 (SSRI Co., Tokyo, Japan) software package for Microsoft Windows. Descriptive statistics for continuous variables, such as component positions, HKA, surgical duration, and clinical scores, are presented as mean \pm standard deviation. Continuous variables among the three groups were compared using one-way analysis of variance. Bonferroni correction was used for comparison of each significant difference among the three groups. Categorical variables, such as outliers, were compared using the chi-square test. A probability $\mathrm{p}$-value of $<0.05$ was considered statistically significant. A previous power analysis indicated that a sample size of at least 22 patients per group was necessary to detect any intergroup difference in the radiographic parameters with an alpha of 0.05 and a power of $80 \%$.

\section{Results}

The postoperative average component alignments in the coronal and sagittal planes and HKA are shown in Table 2. A post hoc test revealed that the CFA in the KA2 group was significantly closer to being perpendicular to the mechanical axis in the coronal plane than in only the CON group, which demonstrates that the results in the coronal plane were significantly more accurate in the KA2 group than in only the CON group. The differences

Table 2. Individual Component Position and Limb Alignment

\begin{tabular}{lrrrc}
\hline \multicolumn{1}{c}{ Characteristic } & \multicolumn{1}{c}{ KA2 } & \multicolumn{1}{c}{ PSI } & \multicolumn{1}{c}{ CON } & p-value \\
\hline Postoperative HKA $\left(^{\circ}\right)$ & $178.9 \pm 1.6$ & $178.5 \pm 2.2$ & $177.6 \pm 2.2$ & $<0.01$ \\
Coronal femoral angle $\left(^{\circ}\right)$ & $89.1 \pm 1.1$ & $88.1 \pm 1.9$ & $87.6 \pm 2.1$ & $<0.01$ \\
Coronal tibial angle $\left(^{\circ}\right)$ & $89.3 \pm 1.6$ & $86.7 \pm 2.6$ & $89.6 \pm 1.8$ & $<0.01$ \\
\hline
\end{tabular}

Values are presented as mean \pm standard deviation.

In the coronal plane, a positive value indicates varus alignment and a negative value indicates valgus alignment on the femoral side. In the sagittal plane, a positive value indicates flexion on the femoral side. KA2: KneeAlign2, PSI: patient-specific instrumentation, CON: conventional intramedullary instrument, HKA: hip-knee-ankle angle.

${ }^{a}$ Statistically significant $(\mathrm{p}<0.05)$. in the coronal plane both between KA2 and PSI groups and between PSI and CON groups were insignificant. In the sagittal plane, the SFA in the PSI group significantly deviated from perpendicular to the distal femoral axis as compared to the KA2 and CON groups. In other words, the PSI group had significantly more flexion than the KA2 and CON groups, thus accuracy in the sagittal plane was significantly better in the KA2 and CON groups than in the PSI group. The difference in the SFA between the KA2 and CON groups was insignificant. There was a significant difference in the postoperative HKA among the three groups. A post-hoc test revealed that there was a significant difference only between KA2 and CON groups.

The percentage of outliers of components that were not located within $3^{\circ}$ from the target is summarized in Table 3 . There were significantly fewer outliers in the coronal plane in the KA2 group. The difference in the number of outliers in the coronal plane between the PSI and CON groups was insignificant. There were

Table 3. Outliers in Femoral Component Positions

\begin{tabular}{lcccr}
\hline \multicolumn{1}{c}{ Characteristic } & KA2 (\%) & PSI (\%) & CON (\%) & p-value \\
\hline $\begin{array}{l}\text { Hip-knee-ankle angle }\left(^{\circ}\right) \\
\text { Coronal plane }\end{array}$ & 9.1 & 22.5 & 28.1 & 0.283 \\
$\quad \begin{array}{l}\text { Femoral component } \\
\text { Sagittal plane }\end{array}$ & 0 & 12.9 & 31.3 & $<0.01^{\text {a) }}$ \\
$\quad$ Femoral component & 9.0 & 37.5 & 0 & $<0.01^{\text {a) }}$ \\
\hline
\end{tabular}

Deviation of $>3^{\circ}$ from the target in any plane was defined as an outlier. KA2: KneeAlign2, PSI: patient-specific instrumentation, CON: conventional intramedullary instrument.

${ }^{a}$ Statistically significant $(\mathrm{p}<0.05)$.

Table 4. Postoperative Characteristics

\begin{tabular}{lcccr}
\hline Characteristic & KA2 & PSI & CON & p-value \\
\hline $\begin{array}{l}\text { Surgical duration } \\
\text { (min) }\end{array}$ & $123.3 \pm 20.8$ & $114.1 \pm 20.6$ & $121.1 \pm 18.3$ & 0.080 \\
$\begin{array}{c}\text { Postoperative } \\
\text { estimated blood }\end{array}$ & $422.5 \pm 187.5$ & $402.2 \pm 189.3$ & $563.6 \pm 261.9$ & $<0.01^{\mathrm{a})}$ \\
$\quad$ & & & & \\
loss (mL) & $122.3 \pm 9.9$ & $125.2 \pm 13.7$ & $123.5 \pm 9.6$ & 0.298 \\
$\begin{array}{l}\text { Postoperative } \\
\text { ROM }\left(^{\circ}\right)\end{array}$ & & & & \\
$\begin{array}{l}\text { Postoperative KSS } \\
\text { Postoperative knee } \\
\text { function score }\end{array}$ & $75.7 \pm 12.3$ & $74.8 \pm 15.2$ & $78.4 \pm 14.1$ & 0.281 \\
\hline
\end{tabular}

Values are presented as mean \pm standard deviation.

KA2: KneeAlign2, PSI: patient-specific instrumentation, CON: conventional intramedullary instrument, ROM: range of motion, KSS: Knee Society score.

${ }^{a}$ Statistically significant $(\mathrm{p}<0.05)$. 
significantly more outliers in the sagittal plane in the PSI group. The difference in the number of outliers in the sagittal plane between the KA2 and CON groups was insignificant.

Regarding postoperative tibial component positions, the mean CTA was $89.8^{\circ} \pm 1.4^{\circ}$ with alignment for $96.8 \%$ of patients within $3^{\circ}$ perpendicular to the coronal mechanical axis of the tibia in the KA2 group, $90.1^{\circ} \pm 1.6^{\circ}$ for $96.7 \%$ of patients in the PSI group, and $90.4^{\circ} \pm 1.9^{\circ}$ for $93.7 \%$ of patients in the CON group. There were no significant differences in the tibial component positions in either the coronal or sagittal plane among the three groups.

Postoperative characteristics are shown in Table 4. There was no significant difference in the mean surgical duration between the KA2, PSI, and CON groups. Also, there were no complications or revision surgeries in any group. Postoperative estimated blood loss was greater in the CON group than in the KA2 and PSI groups $(\mathrm{p}<0.01)$. There was no difference between the KA2 group and the PSI group in blood loss. There were no significant differences in postoperative clinical findings (KSS and ROM) at 1 year after surgery among the three groups.

\section{Discussion}

The most important finding of this study is that the KA2 system was the most useful instrument for TKA in terms of accurate femoral osteotomy and minimal blood loss. The KA2 system was highly accurate in achieving proper femoral component positioning in both the coronal and sagittal planes. In the sagittal plane, PSI was most likely to result in slight flexion of the femoral component, although differences were minimal and there was no impingement between the spine and cam in posterior-stabilized TKA.

The conventional method of using an intramedullary femoral alignment guide to achieve distal femoral resections perpendicular to the mechanical axes is the most popular method, but it has a limited degree of accuracy ${ }^{3-5)}$. Therefore, CAS was developed to improve accuracy in achieving proper implantation. Many studies have revealed increased accuracy in femoral, tibial, and mechanical alignments with use of a large-console CAS as compared to conventional instrumentation ${ }^{10)}$. However, some studies mentioned potential problems associated with large initial startup costs, pin site locations, and large consoles ${ }^{8-11)}$. Therefore, the use of large-console CAS has not become widespread in spite of better accuracy.

The KA2 system is an accelerometer-based portable navigation device for TKA that combines the alignment accuracy of a largeconsole CAS with the ease of use and convenience of conven- tional alignment methods and compensates for the flaws of largeconsole CAS. Nam et al. ${ }^{12)}$ retrospectively compared the accuracy of the KA2 system with an imageless CAS and found statistically significant differences in the proportion of the patients with acceptable lower extremity alignment $(92.5 \%$ vs. $86.3 \%$, respectively) and femoral component alignment ( $94.9 \%$ vs. $92.5 \%$, respectively), while there was no appreciable difference in the alignment of the tibial component (96.25\% vs. $97.5 \%$, respectively $)^{12}$. Huang et al. ${ }^{20)}$ also reported on the accuracy of the KA2 system for tibial and femoral alignments relative to the mechanical axis: the average times for pinning and navigation were 3.6 minutes for the femur and 2.6 minutes for the tibia. They concluded that the KA2 system accurately re-established the mechanical axis without increasing the surgical duration. In our cohort, the KA2 system improved positioning of the components for coronal and sagittal alignments, with results similar to those of Nam et al. ${ }^{12)}$ and Huang et $\mathrm{al}^{20}{ }^{20}$. Poorer results with the conventional method seem to be related to the use of an intramedullary guide, as the point of entry and the direction selected by the surgeon can affect the alignment. The positioning of components for coronal and sagittal alignments was as accurate with the KA2 system as that of previously reported large-console CAS, thereby demonstrating that the KA2 system was a useful instrument for TKA. Additionally, there was no significant difference in the surgical duration between the KA2 system and the CON. Moreover, the estimated blood loss was lower in the KA2 group than in the CON group, and there was no injury to the intramedullary canal.

PSI is relatively new and was developed to aid the surgeon in achieving accurate component alignment and overall limb alignment. However, current studies have reported controversial results regarding component alignment and overall limb alignment. Some reports comparing the accuracy of the PSI to that of the conventional intramedullary method found no differences in the accuracy of postoperative alignment using radiographs ${ }^{16,17)}$. Stronach et al. ${ }^{17)}$ demonstrated that PSI did not improve overall limb alignment with worsening of the tibial slope in comparison to the conventional method. A systematic review by Thienpont et al. ${ }^{13)}$ reported that PSI showed a significant advantage over the conventional method for alignment of the femoral component in the coronal plane, but the results for the alignment of the tibial component were significantly worse with PSI than the conventional method in the coronal and sagittal alignments. They concluded that the use of PSI did not improve the accuracy of component alignment, as compared with the conventional method. On the other hand, other studies reported that the use of PSI significantly improved the component position and overall limb 
alignment, as with the conventional intramedullary method ${ }^{14,15)}$. Anderl et al. ${ }^{15)}$ reported fewer outliers in overall lower limb alignment and component positions with the use of PSI, as compared with the conventional method. $\mathrm{Ng}$ et al. ${ }^{14)}$ compared PSI with the conventional method by means of CT and found that PSI was more accurate for component positioning for tibial coronal alignment and both tibial and femoral rotational alignments. In our study, there was no significant difference in femoral component alignment in the coronal plane between the use of PSI and the conventional method. However, femoral component alignment in the sagittal plane was better in the CON group than in the PSI group. Victor et al. ${ }^{16)}$ also found a wider range of outliers in the sagittal alignment of the femoral component with the use of PSI in comparison to the conventional method, and they mentioned that a small toggle in the sagittal plane irrevocably led to sagittal plane outliers. In our experience, the sagittal position of the PSI femoral guide was difficult to locate accurately because of the femoral guide of the Signature system had a small toggle. If the accuracy of component positioning was the same or even a little less, we would not recommend routine use of PSI because of the extra cost and waiting time.

Both CAS and PSI are widely used and efficient, but relatively few studies have compared PSI with the large-console CAS. Yan et al. ${ }^{21)}$ conducted a comparative study of PSI, large-console CAS, and the conventional instrument and reported that the use of PSI was insignificantly more likely to result in an excessively flexed femoral component, lower limb alignment, and other components in the coronal and sagittal planes, but the mean surgical duration was significantly longer in the large-console CAS group than in the PSI and conventional method groups. Hence, they do not recommend the routine use of PSI.

KA2 is relatively more portable and easier navigation system than large-console CAS navigation systems; therefore, we assumed that the KA2 system would be more useful than PSI or the conventional method. To our knowledge, this is the first comparative study of the KA2 system with PSI and CON. The results of this study revealed that the KA2 system is useful for accurate implantation of femoral components, as compared to the use of PSI or the conventional method. While the cost of the KA2 system is greater than that of the conventional method, it is less expensive than the large-console navigation CAS. In addition, the KA2 system can prevent intramedullary invasion of an intramedullary rod.

We did not use the KA2 instrument for proximal tibial osteotomy. Nonetheless, alignment of the tibial components in this study was as accurate as that reported in previous studies on tibial com- ponent alignment using the KA2 system or $\mathrm{PSI}^{12,14)}$. Therefore, we hesitated to extend the skin incision to adjust the settings of the tibial KA2 instrument, as we thought that the KA2 guide would be advantageous for only distal femoral resection.

There were some limitations to this study. First, this study was a retrospective case series. We chose instruments depending on the period in which the procedure was performed. Potential confounding factors, such as time to treatment and medical comorbidities, were not included in the analysis. However, there were no significant differences in preoperative characteristics among the three groups. Second, we acknowledge the potential influence of a learning curve. Unfortunately, we have no data pertaining to the learning curve required for use of the KA2 and Signature systems. However, there was no significant difference in the mean surgical duration among the three groups, indicating similar familiarity of the operators with both KA2 and Signature instruments. The KA2 device is simple to use because it provides a level of familiarity to surgeons accustomed to using conventional alignment methods ${ }^{12}$. On PSI (Signature), we had never given up using PSI instruments because of uncomfortable fitting in femoral guides. We used both CT- and MRI-based Signature and found a minor difference of fitting. However, it was not difficult to use either the MRI or CT Signature guide. Additionally, there were no differences in femoral alignments between the MRI- and CT-based ones. Third, we evaluated only AP, lateral, and AP entire lower limb postoperative radiographs. Postoperative CT could have been used to evaluate component rotation for more comprehensive understanding of the alignment. However, CT has several disadvantages, such as expense and radiation exposure, so this imaging modality was not routinely employed.

\section{Conclusions}

The portable accelerometer-based KA2 navigation system enables ideal femoral implantation in the coronal and sagittal planes as compared to PSI and the CON.

\section{Conflict of Interest}

No potential conflict of interest relevant to this article was reported.

\section{References}

1. Matsuda S, Kawahara S, Okazaki K, Tashiro Y, Iwamoto Y. Postoperative alignment and ROM affect patient satisfaction 
after TKA. Clin Orthop Relat Res. 2013;471:127-33.

2. Ritter MA, Davis KE, Meding JB, Pierson JL, Berend ME, Malinzak RA. The effect of alignment and BMI on failure of total knee replacement. J Bone Joint Surg Am. 2011;93:158896.

3. Kim SJ, MacDonald M, Hernandez J, Wixson RL. Computer assisted navigation in total knee arthroplasty: improved coronal alignment. J Arthroplasty. 2005;20(7 Suppl 3):12331.

4. Moon YW, Han JH, Lee KH, Jang SW, Seo JG. Clinical outcome of IM-guided total knee arthroplasty with inappropriate femoral resection in coronal plane. Knee Surg Relat Res. 2013;25:19-24.

5. Rosenberger RE, Hoser C, Quirbach S, Attal R, Hennerbichler A, Fink C. Improved accuracy of component alignment with the implementation of image-free navigation in total knee arthroplasty. Knee Surg Sports Traumatol Arthrosc. 2008;16:249-57.

6. Church JS, Scadden JE, Gupta RR, Cokis C, Williams KA, Janes GC. Embolic phenomena during computer-assisted and conventional total knee replacement. J Bone Joint Surg Br. 2007;89:481-5.

7. Duwelius PJ, Huckfeldt R, Mullins RJ, Shiota T, Woll TS, Lindsey KH, Wheeler D. The effects of femoral intramedullary reaming on pulmonary function in a sheep lung model. J Bone Joint Surg Am. 1997;79:194-202.

8. Mizu-uchi H, Matsuda S, Miura H, Okazaki K, Akasaki Y, Iwamoto Y. The evaluation of post-operative alignment in total knee replacement using a CT-based navigation system. J Bone Joint Surg Br. 2008;90:1025-31.

9. Barrett WP, Mason JB, Moskal JT, Dalury DF, Oliashirazi A, Fisher DA. Comparison of radiographic alignment of imageless computer-assisted surgery vs conventional instrumentation in primary total knee arthroplasty. J Arthroplasty. 2011; 26:1273-84.

10. Mason JB, Fehring TK, Estok R, Banel D, Fahrbach K. Metaanalysis of alignment outcomes in computer-assisted total knee arthroplasty surgery. J Arthroplasty. 2007;22:1097-106.

11. Yamagami R, Taketomi S, Inui H, Sanada T, Nakagawa T, Tanaka S. Myositis ossificans after navigated knee surgery: a report of two cases and literature review. Knee. 2016;23:5614.

12. Nam D, Weeks KD, Reinhardt KR, Nawabi DH, Cross MB,
Mayman DJ. Accelerometer-based, portable navigation vs imageless, large-console computer-assisted navigation in total knee arthroplasty: a comparison of radiographic results. J Arthroplasty. 2013;28:255-61.

13. Thienpont E, Schwab PE, Fennema P. A systematic review and meta-analysis of patient-specific instrumentation for improving alignment of the components in total knee replacement. Bone Joint J. 2014;96:1052-61.

14. Ng VY, Arnott L, Li J, Hopkins R, Lewis J, Sutphen S, Nicholson L, Reader D, McShane MA. Comparison of custom to standard TKA instrumentation with computed tomography. Knee Surg Sports Traumatol Arthrosc. 2014;22:1833-42.

15. Anderl W, Pauzenberger L, Kolblinger R, Kiesselbach G, Brandl G, Laky B, Kriegleder B, Heuberer P, Schwameis E. Patient-specific instrumentation improved mechanical alignment, while early clinical outcome was comparable to conventional instrumentation in TKA. Knee Surg Sports Traumatol Arthrosc. 2016;24:102-11.

16. Victor J, Dujardin J, Vandenneucker H, Arnout N, Bellemans J. Patient-specific guides do not improve accuracy in total knee arthroplasty: a prospective randomized controlled trial. Clin Orthop Relat Res. 2014;472:263-71.

17. Stronach BM, Pelt CE, Erickson JA, Peters CL. Patient-specific instrumentation in total knee arthroplasty provides no improvement in component alignment. J Arthroplasty. 2014; 29:1705-8.

18. Steinhaus ME, McLawhorn AS, Richardson SS, Maher P, Mayman DJ. Handheld navigation device and patientspecific cutting guides result in similar coronal alignment for primary total knee arthroplasty: a retrospective matched cohort study. HSS J. 2016;12:224-34.

19. Nadler SB, Hidalgo JH, Bloch T. Prediction of blood volume in normal human adults. Surgery. 1962;51:224-32.

20. Huang EH, Copp SN, Bugbee WD. Accuracy of a handheld accelerometer-based navigation system for femoral and tibial resection in total knee arthroplasty. J Arthroplasty. 2015;30: 1906-10.

21. Yan CH, Chiu KY, Ng FY, Chan PK, Fang CX. Comparison between patient-specific instruments and conventional instruments and computer navigation in total knee arthroplasty: a randomized controlled trial. Knee Surg Sports Traumatol Arthrosc. 2015;23:3637-45. 